\title{
Synthesis of some novel fluoro isoxazolidine and isoxazoline derivatives using $N$-benzyl fluoro nitrone via cycloaddition reaction in ionic liquid
}

\author{
BHASKAR CHAKRABORTY* and GOVINDA PRASAD LUITEL \\ Organic Chemistry Laboratory, Sikkim Government College, Gangtok 737 102, India \\ e-mail: bhaskargtk@yahoo.com
}

MS received 2 March 2013; revised 31 May 2013; accepted 18 June 2013

\begin{abstract}
Butyl-3-methylimidazolium-based ionic liquids are found to accelerate significantly the intermolecular 1,3-dipolar cycloaddition of $N$-benzyl-fluoro nitrone derived in situ from 2,6-difluoro benzaldehyde and $N$-benzylhydroxylamine, with activated alkenes and electron deficient alkynes to afford enhanced rates and improved yields of novel isoxazolidines and isoxazolines.
\end{abstract}

Keywords. $\quad N$-Benzyl fluoro nitrone; cycloaddition reaction; fluoro isoxazolidine and isoxazolines; ionic liquid; 1,3-amino alcohol; aldehyde/ketone synthesis.

\section{Introduction}

The 1,3-dipolar cycloaddition reactions represent a favourite method for the construction of five-membered heterocycles, important frameworks of various natural products. ${ }^{1}$ In particular, the 1,3-dipolar cycloaddition reaction of nitrones with alkenes and alkynes afford isoxazolidines and isoxazolines which are interesting intermediates for the synthesis of $\beta$-amino alcohols and alkaloids. ${ }^{2,3}$ Isoxazolines possess medicinal activities such as antibacterial, anticonvulsant, antibiotic, antitubercular and antifungal activities. ${ }^{4,5}$ Despite their potential utility, many of these procedures require high temperature and prolonged reaction times (drastic experimental conditions) and also suffer from poor regioselectivity, and lack of simplicity. In few cases, the yields and selectivities reported are far from satisfactory due to the occurrence of several side reactions. ${ }^{6}$ In recent times, ionic liquids have emerged as green solvents with desirable properties such as good solvating ability, wide liquidious range, tunable polarity, high thermal stability, negligible vapour pressure and ease of recyclability. ${ }^{7}$ Therefore, classical organic reactions can be performed in these media with great advantages (yield and selectivity) as compared to conventional conditions. They are referred to as 'designer solvents' as their properties such as hydrophilicity, hydrophobicity, Lewis acidity, viscosity and density can be altered by the fine-tuning of parameters such as the choice of organic cation, inorganic anion and the length of alkyl chain attached to an organic cation (figure 1).

*For correspondence
These structural variations offer flexibility to the chemist to devise the most idealized solvent, catering to the needs of any particular process. Since ionic liquids are entirely composed of non-coordinating ions, they can provide an ideal reaction medium for reactions that involve reactive ionic intermediates. Due to the stabilization of charged intermediates by ionic liquids, they can promote unprecedented selectivities and enhanced reaction rates. Consequently, ionic liquids are being used as recyclable solvents for the immobilization of transition metal based catalysts, Lewis acids and enzymes. ${ }^{8}$ As a result of their green credentials and potential to enhance reaction rates and selectivities, ionic liquids are finding increasing applications in organic synthesis ${ }^{9}$ with an ever-increasing quest for exploration of newer reactions in ionic liquids. ${ }^{10}$

It is known that introduction of fluorine atom into specific position of organic molecule may cause significant changes in the stability, lipophilicity and biological activities of the resulting molecules. ${ }^{11}$ This has been attributed to high electronegativity of the halogen, strong C-F bond and similar size of halogen and hydrogen atoms. The presence of a fluoro group due to a low polarizability and high lipophilicity induces a relative metabolic stability and improves the bioavailability of the modified heterocycles compared to its hydrocarbon analogues. ${ }^{12,13}$

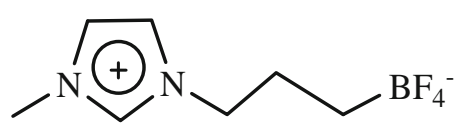

Figure 1. Chemical structure of ionic liquid used in this study. 


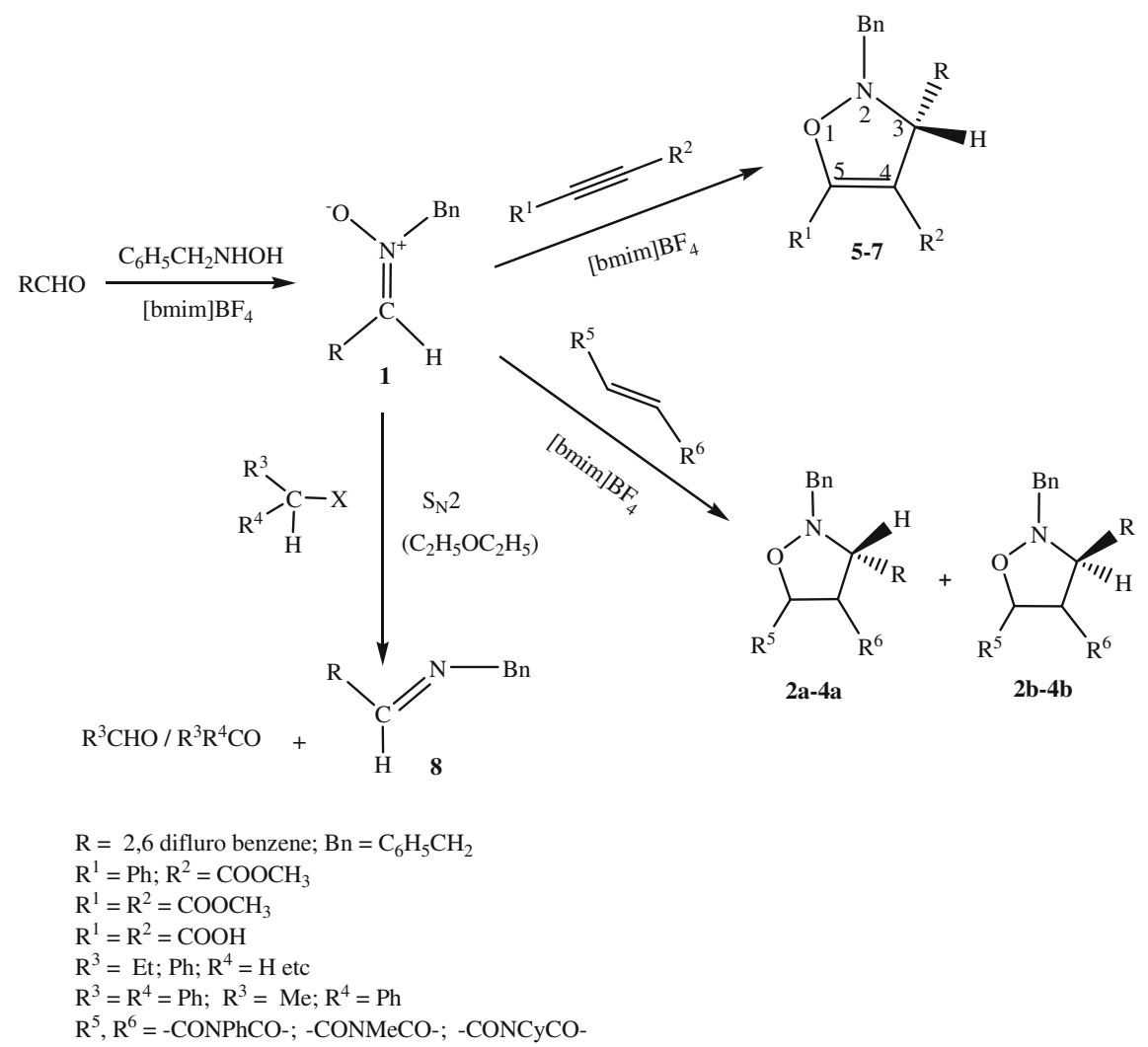

Scheme 1. Synthesis of fluoro isoxazoline and isoxazolidine derivatives using fluoro nitrone and application of the nitrone in atom-efficient aldehyde and ketone synthesis.

In continuation of our effort to establish green methodologies in nitrone cycloaddition reactions, ${ }^{14-18}$ herein, we wish to report the use of ionic liquid as recyclable solvent for 1,3-dipolar cycloaddition reactions of $N$-benzyl fluro nitrone (having vast synthetic potentials) with active alkenes and electron deficient alkynes to produce fluoro isoxazolidine and isoxazoline derivatives with vast biological activity in a one-pot operation (scheme 1, table 1). Compared to conventional conditions, cycloaddition reactions performed in ionic liquids are much faster and selective.

Table 1. Physicochemical data of synthesized compounds $2 \mathbf{a}-\mathbf{4 a} ; \mathbf{2} \mathbf{b}-\mathbf{4 b}$ and $\mathbf{5}-\mathbf{7}$.

\begin{tabular}{|c|c|c|c|c|c|c|}
\hline Entry & Nitrone & Dipolarophile $^{\mathrm{a}}$ & $\begin{array}{l}\text { Time } \\
(\min )\end{array}$ & $\begin{array}{l}\text { Cycloadduct }{ }^{\mathrm{b}} \text {, m.p. }\left({ }^{\circ} \mathrm{C}\right), \\
\mathbf{2} \mathbf{a}-\mathbf{4 a}: \text { cis; } \mathbf{2} \mathbf{b}-\mathbf{4 b} \text { : } \text { trans }^{2}\end{array}$ & $\begin{array}{l}\text { Cis/trans } \\
\text { ratio }(\%)\end{array}$ & Yield $^{\mathrm{c}}(\%)$ \\
\hline 1 & $\begin{array}{l}N \text {-benzyl } \\
\text { fluoro nitrone }\end{array}$ & $\begin{array}{l}N \text {-phenyl } \\
\text { maleimide }\end{array}$ & $\begin{array}{c}26 \\
(12 \mathrm{~h})\end{array}$ & $\begin{array}{l}\text { 2a: White crystals, } 128 \\
\text { 2b: White crystals, } 102\end{array}$ & $\begin{array}{l}\text { 2a: } 66 \\
\text { 2b: } 22\end{array}$ & $88(68)$ \\
\hline 2 & $\begin{array}{l}N \text {-benzyl } \\
\text { fluoro nitrone }\end{array}$ & $\begin{array}{l}N \text {-methyl } \\
\text { maleimide }\end{array}$ & $\begin{array}{c}30 \\
(13 \mathrm{~h})\end{array}$ & $\begin{array}{l}\text { 3a: White solid, } 135 \\
\text { 3b: White solid, } 120\end{array}$ & $\begin{array}{l}\text { 3a: } 65 \\
\text { 3b: } 21\end{array}$ & $86(66)$ \\
\hline 3 & $\begin{array}{l}N \text {-benzyl } \\
\text { fluoro nitrone }\end{array}$ & $\begin{array}{l}N \text {-cyclohexyl } \\
\text { maleimide }\end{array}$ & $\begin{array}{c}36 \\
(13 \mathrm{~h})\end{array}$ & $\begin{array}{l}\text { 4a: Yellow crystals, } 142 \\
\text { 4b: Yellow crystals, } 113\end{array}$ & $\begin{array}{l}\mathbf{4 a}: 63 \\
\mathbf{4 b}: 22\end{array}$ & $85(66)$ \\
\hline 4 & $\begin{array}{l}N \text {-benzyl } \\
\text { fluoro nitrone }\end{array}$ & $\begin{array}{l}\text { Methyl phenyl } \\
\text { propiolate }\end{array}$ & $\begin{array}{c}26 \\
(17 \mathrm{~h})\end{array}$ & 5: Dark red thick liquid & & $88(67)$ \\
\hline 5 & $\begin{array}{l}N \text {-benzyl } \\
\text { fluoro nitrone }\end{array}$ & $\begin{array}{l}\text { Dimethyl acetylene } \\
\text { dicarboxylate }\end{array}$ & $\begin{array}{c}30 \\
(19 \mathrm{~h})\end{array}$ & 6: Red viscous liquid & & $86(65)$ \\
\hline 6 & $\begin{array}{l}N \text {-benzyl } \\
\text { fluoro nitrone }\end{array}$ & $\begin{array}{l}\text { Acetylene } \\
\text { dicarboxylic acid }\end{array}$ & $\begin{array}{c}32 \\
(18 \mathrm{~h})\end{array}$ & 7: Colourless thick liquid & & $86(66)$ \\
\hline
\end{tabular}

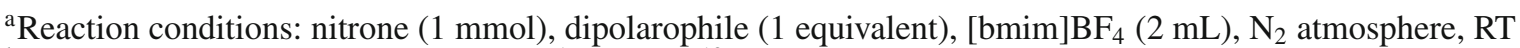

${ }^{\mathrm{b}}$ All products were characterized by IR, ${ }^{1} \mathrm{H}$ NMR, ${ }^{13} \mathrm{C}$ NMR and MS spectral data

${ }^{\mathrm{c}}$ Isolated yield after purification. Figures in parentheses indicate reactions performed by conventional methods 


\section{Experimental}

\subsection{General procedures}

${ }^{1} \mathrm{H}$ nuclear magnetic resonance (NMR) spectra were recorded with a Bruker DRX 300 spectrometer (300 MHz, FT NMR) using tetra methyl silane (TMS) as internal standard. ${ }^{13} \mathrm{C}$ NMR spectra were recorded on the same instrument at $75 \mathrm{MHz}$. The coupling constants $(J)$ are given in Hz. IR spectra were obtained with a Perkin-Elmer RX 1-881 machine as film or as $\mathrm{KBr}$ pellets for all the products. Mass spectroscopy (MS) spectra were recorded with a Jeol SX102 (FAB) instrument. Elemental analyses $(\mathrm{C}, \mathrm{H}, \mathrm{N})$ were performed with a Perkin-Elmer 2400 series CHN analyser. All the reactions were monitored by thin-layer chromatography (TLC) using $0.25 \mathrm{~mm}$ silica gel plates (Merck $60 \mathrm{~F}_{254}$ UV indicator), while column chromatography was performed with silica gel (E Merck India) 60-200 mesh. Starting materials and reagents used in the reactions ( $N$-benzylhydroxylamine, 2,6 difluoro benzaldehyde) were obtained commercially from Aldrich, Lancaster, and were used without purification, unless otherwise indicated. All other reagents and solvents were purified after receiving from commercial suppliers.

2.1a General procedure of synthesis of $N$-benzyl fluoro nitrone (1) in ionic liquid: 2,6-Difluoro benzaldehyde ( $1 \mathrm{mmol}$ ) and $N$-benzylhydroxylamine (1 equivalent) was added to [bmim] $\mathrm{BF}_{4}(2 \mathrm{~mL})$ in a $10 \mathrm{~mL}$ conical flask, mixed thoroughly and stirred at room temperature for $60 \mathrm{~min}$. The formation of nitrone was monitored by TLC $\left(R_{\mathrm{f}}=0.40\right)$. After completion of reaction, the reaction mixture was washed with diethyl ether $(3 \times 10 \mathrm{~mL})$ and the combined ether extract was concentrated in vacuo to obtain nitrone (1) as white crystalline solid (m.p $42^{\circ} \mathrm{C}$, uncorrected). As the nitrone decomposes at room temperature, in situ reactions were performed with alkene and alkynes.

2.1b Spectroscopic data of nitrone 1: UV $\lambda_{\max }$ $238 \mathrm{~nm}$; IR (KBr): $v_{\max } 3025(\mathrm{~m}), 2235$ (m), 1680 (m), $1610(\mathrm{~s}), 1440(\mathrm{~m}), 1154(\mathrm{~m}), 784(\mathrm{~s}) \mathrm{cm}^{-1}$. ${ }^{1} \mathrm{H}$ NMR $\left(\mathrm{CDCl}_{3}\right): \delta$ 7.96-7.79 (m, 3H, $\left.\mathrm{C}_{6} \mathrm{H}_{3} \mathrm{~F}_{2}\right)$, 7.67-7.35 (m, 5H, $\left.\mathrm{C}_{6} \mathrm{H}_{5} \mathrm{CH}_{2}\right), 6.98\left(\mathrm{~s}, 1 \mathrm{H},-\mathrm{CH}=\mathrm{N}^{+}\right)$, $3.37\left(\mathrm{~s}, 2 \mathrm{H}, \mathrm{C}_{6} \mathrm{H}_{5} \mathrm{CH}_{2}\right) .{ }^{13} \mathrm{CNMR}\left(\mathrm{CDCl}_{3}\right): \delta 142.04$ $\left(\mathrm{CH}=\mathrm{N}^{+}\right), 134.80,134.34,134.12,133.93$ (phenyl carbons), 131.60, 130.00, 129.55, 129.46, 128.67, 128.22 (2,6 difluoro phenyl carbons). 2.1c General procedure of synthesis of novel diastereomeric fluoro isoxazolidine derivatives (2-4) in ionic liquid: $\quad N$-phenyl maleimide ( 1 equivalent) was added in situ at the time of development of nitrone $\mathbf{1}$ and the reaction mixture was further stirred at room temperature for an appropriate time (table 1). After completion of reaction, as indicated by TLC $\left(R_{\mathrm{f}}=0.58,0.64\right)$, the reaction mixture was washed with diethyl ether $(3 \times$ $10 \mathrm{~mL}$ ). The combined ether extracts were concentrated in vacuo and the resulting product mixture was directly charged on silica gel column and eluted with a mixture of ethyl acetate:n-hexane (1:8) to afford pure fluoro isoxazolidines $\mathbf{2 a}$ and $\mathbf{2 b}(88 \%$, entry 1 , table 1 , scheme 1). The rest of the viscous ionic liquid was further washed with ether and dried at $80^{\circ} \mathrm{C}$ under reduced pressure to retain its activity in subsequent runs. Same methodology was adopted for the synthesis of other novel fluoro isoxazolidine derivatives (entries 2 and 3).

2.1d 2-Benzyl-3-(2,6-difluorophenyl)-dihydro-5phenyl-2H-pyrrolo[3,4-d] isoxazole-4,6(5H,6 a-H)dione (2a): White crystals. Yield 66\%; $R_{\mathrm{f}}=0.58$; IR (KBr): $v_{\max } 3020(\mathrm{~m}), 2920(\mathrm{~m}), 2835(\mathrm{~m}), 1758(\mathrm{~s})$, 1690 (s), 1480 (m), 1346 (m), 805 (s), 770 (s) cm ${ }^{-1} ;{ }^{1} \mathrm{H}$ NMR $\left(\mathrm{CDCl}_{3}\right): \delta 7.74-7.68\left(\mathrm{~m}, 3 \mathrm{H}, \mathrm{C}_{6} \mathrm{H}_{3} \mathrm{~F}_{2}\right), 7.12-$ $6.83\left(\mathrm{~m}, 2 \times 5 \mathrm{H}, \mathrm{C}_{6} \mathrm{H}_{5}\right.$ protons $), 5.84(\mathrm{~d}, 1 \mathrm{H}, J=$ $\left.6.70 \mathrm{~Hz}, \mathrm{C}_{5} \mathrm{H}\right), 3.40\left(\mathrm{dd}, 1 \mathrm{H}, J=6.06,6.18 \mathrm{~Hz}, \mathrm{C}_{4} \mathrm{H}\right)$, $3.54\left(\mathrm{~s}, 2 \mathrm{H}, \mathrm{C}_{6} \mathrm{H}_{5} \mathrm{CH}_{2}\right), 2.95(\mathrm{~d}, 1 \mathrm{H}, J=6.32 \mathrm{~Hz}$, $\left.\mathrm{C}_{3} \mathrm{H}\right) ;{ }^{13} \mathrm{C} \mathrm{NMR}\left(\mathrm{CDCl}_{3}\right): \delta 173.42,173.10$ (carbonyl carbons), 138.10, 138.06, 138.02, 137.97, 136.86, 136.81, 136.78, 136.75 (phenyl carbons), 134.34, 134.14, 134.06, 133.76, 133.65 (2,6 difluoro phenyl carbons), $85.22\left(\mathrm{C}_{5}\right), 77.20\left(\mathrm{C}_{3}\right), 58.46\left(\mathrm{C}_{4}\right), 39.55$ $\left(\mathrm{CH}_{2} \mathrm{C}_{6} \mathrm{H}_{5}\right)$; FAB-MS: $m / z 420\left(\mathrm{M}^{+}, 100 \%\right), 343,329$, 306, 252, 216 (B.P.), 113, 91, 77; Anal. Calcd. for $\mathrm{C}_{24} \mathrm{H}_{18} \mathrm{O}_{3} \mathrm{~N}_{2} \mathrm{~F}_{2}$ : C, 68.57; H, 4.28; N, $6.66 \%$. Found: C, 68.44; H, 4.19; N, 6.52.

2.1e 2-Benzyl-3-(2,6-difluorophenyl)-dihydro-5phenyl-2H-pyrrolo[3,4-d] isoxazole-4,6(5H,6 a-H)dione $(2 \mathrm{~b})$ : White crystals. Yield $22 \% ; R_{\mathrm{f}}=0.64$; IR (KBr): $v_{\max } 3010(\mathrm{~m}), 2915(\mathrm{~m}), 2830(\mathrm{~m}), 1764$ (s), $1685(\mathrm{~s}), 1486(\mathrm{~m}), 1340(\mathrm{~m}), 864(\mathrm{~s}), 783$ (s) $\mathrm{cm}^{-1} ;{ }^{1} \mathrm{H} \mathrm{NMR}\left(\mathrm{CDCl}_{3}\right): \delta 7.70-7.66\left(\mathrm{~m}, 3 \mathrm{H}, \mathrm{C}_{6} \mathrm{H}_{3} \mathrm{~F}_{2}\right)$, 7.30-7.12 (m, $2 \times 5 \mathrm{H}, \mathrm{C}_{6} \mathrm{H}_{5}$ protons $), 5.76(\mathrm{~d}, 1 \mathrm{H}$, $\left.J=2.24 \mathrm{~Hz}, \mathrm{C}_{5} \mathrm{H}\right), 3.63(\mathrm{dd}, 1 \mathrm{H}, J=2.26,2.08 \mathrm{~Hz}$, $\left.\mathrm{C}_{4} \mathrm{H}\right), 3.28\left(\mathrm{~s}, 2 \mathrm{H}, \mathrm{C}_{6} \mathrm{H}_{5} \mathrm{CH}_{2}\right), 3.06(\mathrm{~d}, 1 \mathrm{H}, J=$ $\left.3.04 \mathrm{~Hz}, \mathrm{C}_{3} \mathrm{H}\right) ;{ }^{13} \mathrm{C} \mathrm{NMR}\left(\mathrm{CDCl}_{3}\right): \delta 172.40,172.24$ (carbonyl carbons), 137.80, 137.74, 137.72, 137.57, 137.36, 136.34, 136.26, 136.18 (phenyl carbons), 134.80, 134.60, 134.44, 134.22, 134.13 (2,6 difluoro phenyl carbons), $80.65\left(\mathrm{C}_{5}\right), 76.52\left(\mathrm{C}_{3}\right), 57.90\left(\mathrm{C}_{4}\right)$, 
$41.24\left(\mathrm{CH}_{2} \mathrm{C}_{6} \mathrm{H}_{5}\right)$; FAB-MS: $m / z 420\left(\mathrm{M}^{+}, 100 \%\right)$, 343, 329, 306, 216 (B.P.), 113, 91, 77; Anal. Calcd. for $\mathrm{C}_{24} \mathrm{H}_{18} \mathrm{O}_{3} \mathrm{~N}_{2} \mathrm{~F}_{2}$ : C, 68.57; H, 4.28; N, 6.66\%. Found: $\mathrm{C}$, 68.49; H, 4.17; N, 6.50 .

2.1f 2-Benzyl-3-(2,6-difluorophenyl)-dihydro-5methyl-2H-pyrrolo[3,4-d]isoxazole-4,6(5H,6a-H)dione (3a): White solid. Yield 65\%; $R_{\mathrm{f}}=0.54$; IR $(\mathrm{KBr}): v_{\max } 3005(\mathrm{~m}), 2935(\mathrm{~m}), 2820(\mathrm{~m}), 1760(\mathrm{~s})$, 1675 (s), 1465 (s), 1340 (m), 814 (s), 778 (s) cm ${ }^{-1} ;{ }^{1} \mathrm{H}$ NMR $\left(\mathrm{CDCl}_{3}\right): \delta$ 7.89-7.86 $\left(\mathrm{m}, 3 \mathrm{H}, \mathrm{C}_{6} \mathrm{H}_{3} \mathrm{~F}_{2}\right), 7.64$ $7.46\left(\mathrm{~m}, 5 \mathrm{H}, \mathrm{C}_{6} \mathrm{H}_{5}\right.$ protons $), 6.56(\mathrm{~d}, 1 \mathrm{H}, J=6.10 \mathrm{~Hz}$, $\left.\mathrm{C}_{5} \mathrm{H}\right), 3.89$ (s, $2 \mathrm{H}, \mathrm{C}_{6} \mathrm{H}_{5} \mathrm{CH}_{2}$ ), 3.79 (dd, $1 \mathrm{H}, J=6.00$, $\left.5.90 \mathrm{~Hz}, \mathrm{C}_{4} \mathrm{H}\right), 3.49\left(\mathrm{~s}, 3 \mathrm{H}, \mathrm{N}-\mathrm{CH}_{3}\right), 2.95(\mathrm{~d}, 1 \mathrm{H}, J=$ $\left.6.76 \mathrm{~Hz}, \mathrm{C}_{3} \mathrm{H}\right) ;{ }^{13} \mathrm{C} \mathrm{NMR}\left(\mathrm{CDCl}_{3}\right): \delta 170.58,170.50$ (carbonyl carbons), 136.44, 136.40, 136.32, 136.25 (phenyl carbons), 132.70, 132.64, 132.51, 132.43, 132.18 (2,6 difluoro phenyl carbons), $82.98\left(\mathrm{C}_{5}\right), 76.66$ $\left(\mathrm{C}_{3}\right), 59.70\left(\mathrm{C}_{4}\right), 39.60\left(\mathrm{CH}_{2} \mathrm{C}_{6} \mathrm{H}_{5}\right), 37.54\left(\mathrm{~N}-\mathrm{CH}_{3}\right)$; FAB-MS: $m / z 358\left(\mathrm{M}^{+}, 100 \%\right), 345,267,252,244$, 154 (B.P.), 113, 91; Anal. Calcd. for $\mathrm{C}_{19} \mathrm{H}_{16} \mathrm{O}_{3} \mathrm{~N}_{2} \mathrm{~F}_{2}$ : C, 63.68; H, 4.46; N, 7.82\%. Found: C, 63.49; H, 4.36; $\mathrm{N}, 7.57$.

2.1g 2-Benzyl-3-(2,6-difluorophenyl)-dihydro-5methyl-2H-pyrrolo[3,4-d]isoxazole-4,6(5H,6 a-H)dione (3b): White solid. Yield 21\%; $R_{\mathrm{f}}=0.60 ; \mathrm{IR}$ (KBr): $v_{\max } 3015(\mathrm{~m}), 2905(\mathrm{~m}), 2828$ (s), 1760 (s), 1680 (s), 1460 (s), 1355 (m), 820 (s), 783 (s) cm ${ }^{-1} ;{ }^{1} \mathrm{H}$ NMR $\left(\mathrm{CDCl}_{3}\right): \delta$ 7.88-7.84 $\left(\mathrm{m}, 3 \mathrm{H}, \mathrm{C}_{6} \mathrm{H}_{3} \mathrm{~F}_{2}\right), 7.60$ $7.49\left(\mathrm{~m}, 5 \mathrm{H}, \mathrm{C}_{6} \mathrm{H}_{5}\right.$ protons), $6.52(\mathrm{~d}, 1 \mathrm{H}, J=3.22 \mathrm{~Hz}$, $\left.\mathrm{C}_{5} \mathrm{H}\right), 3.84$ (s, $\left.2 \mathrm{H}, \mathrm{C}_{6} \mathrm{H}_{5} \mathrm{CH}_{2}\right), 3.76(\mathrm{dd}, 1 \mathrm{H}, J=1.96$, $\left.2.12 \mathrm{~Hz}, \mathrm{C}_{4} \mathrm{H}\right), 3.47\left(\mathrm{~s}, 3 \mathrm{H}, \mathrm{N}-\mathrm{CH}_{3}\right), 2.96(\mathrm{~d}, 1 \mathrm{H}, J=$ $\left.1.96 \mathrm{~Hz}, \mathrm{C}_{3} \mathrm{H}\right) ;{ }^{13} \mathrm{C} \mathrm{NMR}\left(\mathrm{CDCl}_{3}\right): \delta 171.34,171.27$ (carbonyl carbons), 135.98, 135.94, 135.82, 135.75 (phenyl carbons), 133.12, 133.04, 132.91, 132.83, 132.77 (2,6 difluoro phenyl carbons), $84.08\left(\mathrm{C}_{5}\right), 73.80$ $\left(\mathrm{C}_{3}\right), 54.95\left(\mathrm{C}_{4}\right), 41.42\left(\mathrm{CH}_{2} \mathrm{C}_{6} \mathrm{H}_{5}\right), 39.05\left(\mathrm{~N}-\mathrm{CH}_{3}\right)$; FAB-MS: $m / z 358\left(\mathrm{M}^{+}, 100 \%\right), 345,267,252,154$ (B.P.), 113, 91, 77; Anal. Calcd. for $\mathrm{C}_{19} \mathrm{H}_{16} \mathrm{O}_{3} \mathrm{~N}_{2} \mathrm{~F}_{2}$ : C, 63.68; H, 4.46; N, 7.82\%. Found: C, 63.42; H, 4.32; N, 7.62 .

2.1h 2-Benzyl-5-cyclohexyl-3-(2,6-difluorophenyl)dihydro-2H-pyrrolo[3,4-d]isoxazole-4,6(5H,6 a-H)dione (4a): Yellow crystals. Yield $63 \%, R_{\mathrm{f}}=0.50$; IR (KBr): $v_{\max } 3015(\mathrm{~m}), 2900$ (s), 2840 (m), 1760 (s), 1674 (br, s), 1470 (s), 1330 (m), 805 (s), 786 (s) $\mathrm{cm}^{-1} ;{ }^{1} \mathrm{H}$ NMR $\left(\mathrm{CDCl}_{3}\right): \delta 7.60-7.56\left(\mathrm{~m}, 3 \mathrm{H}, \mathrm{C}_{6} \mathrm{H}_{3} \mathrm{~F}_{2}\right)$, 7.17-7.06 (m, 5H, $\mathrm{C}_{6} \mathrm{H}_{5}$ protons $), 6.30(\mathrm{~d}, 1 \mathrm{H}, J=$ $\left.6.74 \mathrm{~Hz}, \mathrm{C}_{5} \mathrm{H}\right), 3.60\left(\mathrm{~s}, 2 \mathrm{H}, \mathrm{C}_{6} \mathrm{H}_{5} \mathrm{CH}_{2}\right), 3.42(\mathrm{dd}, 1 \mathrm{H}$,
$\left.J=6.20,6.10 \mathrm{~Hz}, \mathrm{C}_{4} \mathrm{H}\right), 2.83(\mathrm{~d}, 1 \mathrm{H}, J=6.76 \mathrm{~Hz}$, $\left.\mathrm{C}_{3} \mathrm{H}\right), 1.95-1.52\left(\mathrm{~m}, 11 \mathrm{H}\right.$, cyclohexyl protons); ${ }^{13} \mathrm{C}$ NMR $\left(\mathrm{CDCl}_{3}\right): \delta 168.54,168.50$ (carbonyl carbons), 131.66, 131.60, 131.55, 131.50 (phenyl carbons), $129.15,129.06,128.80,128.73,128.68$ (2,6 difluoro phenyl carbons), $83.60\left(\mathrm{C}_{5}\right), 74.55\left(\mathrm{C}_{3}\right), 58.24$ $\left(\mathrm{C}_{4}\right), 38.78\left(\mathrm{CH}_{2} \mathrm{C}_{6} \mathrm{H}_{5}\right), 27.40,27.29,26.87,26.70$, 26.58, 26.46 (cyclohexyl carbons); FAB-MS: $m / z 426$ $\left(\mathrm{M}^{+}, 100 \%\right), 343,335,312,252$, 222 (B.P.), 113, 91, 83; Anal. Calcd. for $\mathrm{C}_{24} \mathrm{H}_{24} \mathrm{O}_{3} \mathrm{~N}_{2} \mathrm{~F}_{2}$ : C, 67.60; H, 5.63; $\mathrm{N}, 6.57 \%$. Found: C, 67.46; H, 5.35; N, 6.37.

2.1i 2-Benzyl-5-cyclohexyl-3-(2,6-difluorophenyl)dihydro-2H-pyrrolo[3,4-d]isoxazole-4,6(5H,6 a-H)dione $(4 \mathrm{~b})$ : Yellow crystals. Yield $22 \%, R_{\mathrm{f}}=0.62$; IR (KBr): $v_{\max } 3010(\mathrm{~m}), 2905(\mathrm{~s}), 2835(\mathrm{~m}), 1764(\mathrm{~s})$, 1675 (s), 1466 (s), 1336 (m), 815 (s), 783 (s) cm ${ }^{-1} ;{ }^{1} \mathrm{H}$ NMR $\left(\mathrm{CDCl}_{3}\right): \delta 7.52-7.85\left(\mathrm{~m}, 3 \mathrm{H}, \mathrm{C}_{6} \mathrm{H}_{3} \mathrm{~F}_{2}\right), 7.25-$ $7.14\left(\mathrm{~m}, 5 \mathrm{H}, \mathrm{C}_{6} \mathrm{H}_{5}\right.$ protons), $6.14(\mathrm{~d}, 1 \mathrm{H}, J=1.88 \mathrm{~Hz}$, $\left.\mathrm{C}_{5} \mathrm{H}\right), 3.55\left(\mathrm{~s}, 2 \mathrm{H}, \mathrm{C}_{6} \mathrm{H}_{5} \mathrm{CH}_{2}\right), 3.38(\mathrm{dd}, 1 \mathrm{H}, J=2.08$, $\left.2.04 \mathrm{~Hz}, \mathrm{C}_{4} \mathrm{H}\right), 2.80\left(\mathrm{~d}, 1 \mathrm{H}, J=1.80 \mathrm{~Hz}, \mathrm{C}_{3} \mathrm{H}\right), 1.90$ 1.38 (m, $11 \mathrm{H}$, cyclohexyl protons); ${ }^{13} \mathrm{C} \mathrm{NMR}\left(\mathrm{CDCl}_{3}\right)$ : $\delta$ 169.88, 169.83 (carbonyl carbons), 130.54, 130.49, 130.45 , 130.32 (phenyl carbons), 128.77, 128.68, 128.56, 128.53, 128.48 (2,6 difluoro phenyl carbons), $80.44\left(\mathrm{C}_{5}\right), 77.50\left(\mathrm{C}_{3}\right), 58.97\left(\mathrm{C}_{4}\right), 37.05\left(\mathrm{CH}_{2} \mathrm{C}_{6} \mathrm{H}_{5}\right)$, 25.30, 25.22, 25.17, 25.06, 24.88, 24.76 (cyclohexyl carbons); FAB-MS: $m / z 426\left(\mathrm{M}^{+}, 100 \%\right), 343,312$, 252, 222 (B.P.), 113, 91, 83, 77; Anal. Calcd. for $\mathrm{C}_{24} \mathrm{H}_{24} \mathrm{O}_{3} \mathrm{~N}_{2} \mathrm{~F}_{2}$ : C, 67.60; H, 5.63; N, 6.57\%. Found: C, 67.37; H, 5.40; N, 6.33.

\section{$2.1 \mathrm{j}$ General procedure of synthesis of novel fluoro} isoxazoline derivatives (5-7) in ionic liquid: Methyl phenyl propiolate (1 equivalent) was added in situ at the time of development of nitrone $\mathbf{1}$ and the reaction mixture was further stirred at room temperature for an appropriate time (table 1). After completion of reaction, as indicated by TLC $\left(R_{\mathrm{f}}=0.66\right)$, the reaction mixture was washed with diethyl ether $(3 \times 10 \mathrm{~mL})$. The combined ether extract was concentrated in vacuo and the resulting crude product was directly charged on silica gel column and eluted with a mixture of ethyl acetate: $n$ hexane (1:8) to afford pure fluoro isoxazoline 5 (88\%, entry 4 , table 1 , scheme 1 ). The rest of the viscous ionic liquid was further washed with ether and dried at $80^{\circ} \mathrm{C}$ under reduced pressure to retain its activity in subsequent runs. Same methodology was adopted for the synthesis of other novel fluoro isoxazoline derivatives (entries 5 and 6). 
2.2 Methyl-2-benzyl-3-(2,6-difluorophenyl-2,3dihydro-5-phenylisoxazole-4-carboxylate (5)

Dark red thick liquid. Yield 88\%; $R_{\mathrm{f}}=0.66$; IR $(\mathrm{KBr})$ : $v_{\max } 3010(\mathrm{~m}), 2246(\mathrm{~m}), 1740(\mathrm{~s}), 1710$ (s), 1690 (s), 1610 (s), 1480 (s), 1324 (s), 1215 (s), 810 (m), $782(\mathrm{~s}) \mathrm{cm}^{-1} ;{ }^{1} \mathrm{H} \mathrm{NMR}\left(\mathrm{CDCl}_{3}\right): \delta 7.87-7.80(\mathrm{~m}, 3 \mathrm{H}$, $\left.\mathrm{C}_{6} \mathrm{H}_{3} \mathrm{~F}_{2}\right), 7.68-7.31\left(\mathrm{~m}, 2 \times 5 \mathrm{H}, \mathrm{C}_{6} \mathrm{H}_{5}\right), 3.38(\mathrm{~s}, 3 \mathrm{H}$, $\left.-\mathrm{COOCH}_{3}\right), 2.68\left(\mathrm{~s}, 2 \mathrm{H}, \mathrm{C}_{6} \mathrm{H}_{5} \mathrm{CH}_{2}\right), 1.25\left(\mathrm{~s}, 1 \mathrm{H}, \mathrm{C}_{3} \mathrm{H}\right)$; ${ }^{13} \mathrm{C} \mathrm{NMR}\left(\mathrm{CDCl}_{3}\right): \delta 168.52\left(-\mathrm{COOCH}_{3}\right), 137.20$, 137.04, 136.87, 136.66, 135.65, 135.48, 135.20, 134.93 (aromatic carbons), 132.77, 132.35, 132.08, 131.78, 130.80, 129.90 (2,6 difluoro phenyl carbons), 88.16 $\left(\mathrm{C}_{5}\right), 73.60\left(\mathrm{C}_{3}\right), 58.45\left(\mathrm{C}_{4}\right), 45.17\left(-\mathrm{COOCH}_{3}\right), 36.80$

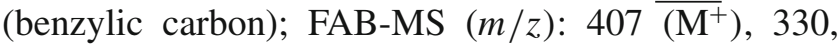
294, 211 (B.P.), 203, 113, 105, 91, 77. Anal. Calcd. for $\mathrm{C}_{24} \mathrm{H}_{19} \mathrm{O}_{3} \mathrm{~F}_{2} \mathrm{~N}$ : C, 70.76; H, 4.66; N, 3.43. Found: C, $70.63 ; \mathrm{H}, 4.61 ; \mathrm{N}, 3.35 \%$.

2.2a Dimethyl-2-benzyl-3-(2,6-difluorophenyl)-2,3dihydroisoxazole-4,5-dicarboxylate (6): Red viscous liquid. Yield 86\%; $R_{\mathrm{f}}=0.60 ; \mathrm{IR}(\mathrm{KBr}): v_{\max } 3015$ (m), $2250(\mathrm{~m}), 1725$ (s), 1685 (s), 1610 (s), 1440 (s), 1260 (s), 1225 (s), $805(\mathrm{~m}), 780(\mathrm{~s}) \mathrm{cm}^{-1} ;{ }^{1} \mathrm{H}$ NMR $\left(\mathrm{CDCl}_{3}\right): \delta$ 7.44-7.36 (m, 3H, $\left.\mathrm{C}_{6} \mathrm{H}_{3} \mathrm{~F}_{2}\right), 7.10-6.98$ $\left(\mathrm{m}, 5 \mathrm{H}, \mathrm{C}_{6} \mathrm{H}_{5}\right), 3.30\left(\mathrm{~s}, 3 \mathrm{H},-\mathrm{COOCH}_{3}\right), 3.24(\mathrm{~s}, 3 \mathrm{H}$, $\left.-\mathrm{COOCH}_{3}\right), 2.55\left(\mathrm{~s}, 2 \mathrm{H}, \mathrm{C}_{6} \mathrm{H}_{5} \mathrm{CH}_{2}\right), 1.72\left(\mathrm{~s}, 1 \mathrm{H}, \mathrm{C}_{3} \mathrm{H}\right)$; ${ }^{13} \mathrm{C} \mathrm{NMR}\left(\mathrm{CDCl}_{3}\right): \delta$ 169.74, $169.58\left(-\mathrm{COOCH}_{3}\right.$, carbonyl carbons of the ester group), 135.80, 135.73, 135.54, 135.47 (aromatic carbons), 133.30, 133.28, 133.24, 133.15, 133.12, 133.05 (2,6 difluoro phenyl carbons $), 85.25\left(\mathrm{C}_{5}\right), 77.80\left(\mathrm{C}_{3}\right), 56.90\left(\mathrm{C}_{4}\right), 45.74$, $44.82\left(-\mathrm{COOCH}_{3}\right.$, methyl carbons of the ester methyl group), 39.23 (benzylic carbon); FAB-MS $(\mathrm{m} / \mathrm{z}): 389$ $\left(\mathrm{M}^{+}\right)$, 358, 330, 302, 276, 271 (B.P.), 185, 113, 91, 77; Anal. Calcd. for $\mathrm{C}_{20} \mathrm{H}_{17} \mathrm{O}_{5} \mathrm{~F}_{2} \mathrm{~N}$ : C, 61.69; H, 4.37; N, 3.59. Found: C, $61.58 ; \mathrm{H}, 4.26 ; \mathrm{N}, 3.35 \%$.

2.2b 2-Benzyl-3-(2,6-difluorophenyl)-2,3-dihydroisoxazole-4,5-dicarboxylic acid (7): Colourless thick liquid. Yield $66 \% ; R_{\mathrm{f}}=0.66 ; \mathrm{IR}(\mathrm{KBr}): v_{\max } 3010$ (m), 2995 (br), 2246 (m), 1760 (s), 1610 (s), 1480 (s), 1324 (s), 1215 (s), 1105 (s), 800 (m), 782 (s) cm ${ }^{-1} ;{ }^{1} \mathrm{H}$ NMR $\left(\mathrm{CDCl}_{3}\right): \delta 10.02(\mathrm{~s}, 2 \mathrm{H}, 2 \mathrm{XCOOH}), 7.90-7.87$ $\left(\mathrm{m}, 3 \mathrm{H}, \mathrm{C}_{6} \mathrm{H}_{3} \mathrm{~F}_{2}\right), 7.66-7.44\left(\mathrm{~m}, 5 \mathrm{H}, \mathrm{C}_{6} \mathrm{H}_{5}\right), 2.91(\mathrm{~s}$, $\left.2 \mathrm{H}, \mathrm{C}_{6} \mathrm{H}_{5} \mathrm{CH}_{2}\right), 2.88\left(\mathrm{~s}, 1 \mathrm{H}, \mathrm{C}_{3} \mathrm{H}\right) ;{ }^{13} \mathrm{C} \mathrm{NMR}\left(\mathrm{CDCl}_{3}\right)$ : $\delta$ 173.69, 172.04 (carboxyl carbons), 138.50, 138.44, 138.37, 138.26 (aromatic carbons), 135.44, 135.40, 135.28, 134.93, 134.87, 134.75 (2,6 difluoro phenyl carbons), $88.20\left(\mathrm{C}_{5}\right), 74.43\left(\mathrm{C}_{3}\right), 58.60\left(\mathrm{C}_{4}\right), 37.87$ (benzylic carbon); FAB-MS $(m / z): 361\left(\mathbf{M}^{+}\right), 344$, 316, 288, 271 (B.P.), 248, 157, 113, 91, 77. Anal.
Calcd. for $\mathrm{C}_{18} \mathrm{H}_{13} \mathrm{O}_{5} \mathrm{~F}_{2} \mathrm{~N}$ : C, 59.83; H, 3.60; N, 3.87. Found: C, 59.75; H, 3.40; N, 3.58\%.

\section{Results and discussion}

As an example, the reaction between 1 and alkynes, afforded cycloaddition derivative 5 after $17 \mathrm{~h}$ in $\mathrm{CH}_{2} \mathrm{Cl}_{2}$ in $67 \%$ yield and $88 \%$ yield (entry 4 ) in $\left[\mathrm{bmim}^{\mathrm{B}} \mathrm{BF}_{4}\right.$ at room temperature after $26 \mathrm{~min}$, respectively. In a typical procedure, $1 \mathrm{mmol}$ of nitrone was mixed with 1 equivalent of alkynes/alkenes in $[\mathrm{bmim}] \mathrm{BF}_{4}(2 \mathrm{~mL})$ under stirring, at room temperature. After the development of nitrone (monitored by TLC), $1 \mathrm{mmol}$ of dipolarophile was added in situ and progress of the reaction was monitored by TLC. After completion of reaction, the reaction mixture was washed with diethyl ether $(3 \times$ $10 \mathrm{~mL}$ ). The combined ether extracts were concentrated in vacuo and the resulting product was directly charged on silica gel column and eluted with a mixture of ethyl acetate: $n$-hexane (1:8) to afford pure isoxazoline. The rest of the viscous ionic liquid was further washed with diethyl ether and dried at $80^{\circ} \mathrm{C}$ under reduced pressure to retain its activity in subsequent runs and was reused up to five times without loss of activity nor selectivity after five cycles. We have intentionally stopped the recycle at the fifth cycle, however we are convinced that this process may be carried out many more times. Excellent diastereofacial selectivity and faster reaction reaction rates have been observed when the reaction of nitrone $\mathbf{1}$ with activated alkenes (maleimides) are carried out in room temperature ionic liquids (RTILs). For example, the reaction between 1 with $N$-phenyl maleimide, afforded cycloaddition derivatives $\mathbf{2 a}$ and 2b after $12 \mathrm{~h}$ in $\mathrm{CH}_{2} \mathrm{Cl}_{2}$ in $68 \%$ yield and $88 \%$ yield (entry 1) in $[\mathrm{bmim}] \mathrm{BF}_{4}$ at room temperature after $26 \mathrm{~min}$, respectively. The addition of nitrone $\mathbf{1}$ to maleimides results in a mixture of diastereomer $\mathbf{2 a - 4 a}$ and $\mathbf{2 b}-\mathbf{4 b}$ (almost 65:25 ratio in all cases) and generation of as many as three chiral centres in a single step. Studies of organic reactions in ionic liquid show

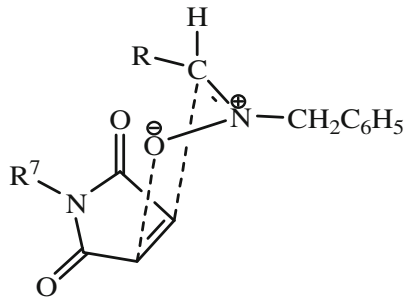

TS 1

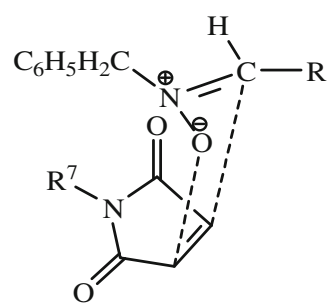

TS 2
$\mathrm{R}^{7}=\mathrm{CH}_{3} / \mathrm{C}_{6} \mathrm{H}_{5} / \mathrm{C}_{6} \mathrm{H}_{11}$

Figure 2. Exo/endo approach of nitrone to maleimides in cycloaddition reactions. 

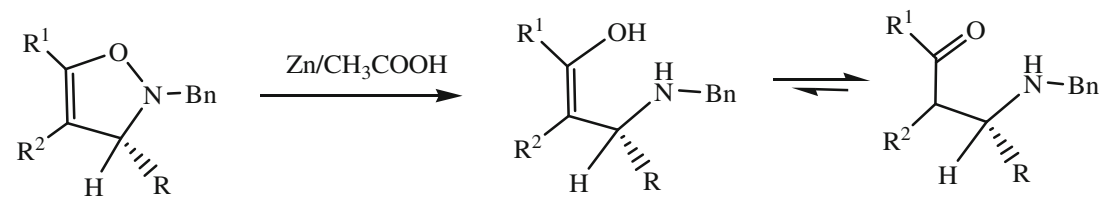

Scheme 2. Synthesis of 1,3 amino alcohol from isoxazoline derivatives.

that there is a higher probability of the formation of mixture of diastereomers when ionic liquid is used as solvent rather than conventional organic solvents. These results can be rationalized by an exo approach of nitrone 1, which has Zconfiguration for the formation of major cycloadducts $\mathbf{2 a - 4 a}$ (transition state 1, figure 2). ${ }^{19}$ Minor cycloadducts $\mathbf{2 b}-\mathbf{4 b}$ are formed by the endo approach of $Z$ nitrone (transition state 2 , figure 2). ${ }^{19}$ The mixture of diastereomers is identified by considering the multiplicity of the proton signals at 3-H and 4-H along with their coupling constant values. ${ }^{20,21}$ The most significant differences in the ${ }^{1} \mathrm{H}$ NMR data for the diastereomers is the position and multiplicity of the $3-\mathrm{H}$ signal. In the major adducts $\mathbf{2 a}-$ 4a, coupling constant between $3-\mathrm{H}$ and $4-\mathrm{H}$ has been measured as $J_{3,4} \sim 6.26 \mathrm{~Hz}$ implying a cis relationship between $\mathrm{H}-3$ and $\mathrm{H}-4$, while for minor adducts $\mathbf{2 b}-\mathbf{4} \mathbf{b}$, $J_{3,4}$ is $\sim 2.26 \mathrm{~Hz}$ implies a trans relationship between $\mathrm{H}-3$ and H-4. ${ }^{20,21}$

Several butylmethylimidazolium based ionic liquids (ILs), [bmim]X, with varying anions $\left(\mathrm{X}=\mathrm{PF}_{6}^{-}\right.$, $\mathrm{Br}^{-}, \mathrm{BF}_{4}^{-}$) were screened for this reaction. Evidently, [bmim] $\mathrm{BF}_{4}$ was found to be superior in terms of yield $(88 \%)$ and reaction time $(26 \mathrm{~min})$ as compared with [bmim] $\mathrm{PF}_{6}(84 \%$; $43 \mathrm{~min}$; entry 4$)$. For optimizing conditions, we used the substrates in different ratios. It was found that best results were obtained using 1:1 reactant ratio. The reaction in $[\mathrm{bmim}] \mathrm{BF}_{4}$ was also conducted at elevated temperatures for optimizing the conditions and no significant improvements were observed in yields and reaction times. We examined the reaction under neat condition also, without using IL, to demonstrate catalytic ability of [bmim] $\mathrm{BF}_{4}$. This result clearly indicates that $[\mathrm{bmim}] \mathrm{BF}_{4}$ has significant catalytic role in this reaction (table 1).

All the novel fluoro cycloadducts are stable and prominent molecular ion peak, base peaks are obtained in the mass spectrum as expected. In case of fluoro isoxazoline derivatives (5-7), we have also obtained expected fragmentation peaks due to the development of different aziridine derivatives. Base peaks are obtained due to loss of $\mathrm{PhCO}$ for phenyl methyl propiolate, $\mathrm{COOCH}_{3}$ for dimethyl acetylene dicarboxylate and $\mathrm{COOH}$ for acetylene dicarboxylic acid cycloadducts, respectively. Hence, it is confirmed that during mass fragmentation, the isoxazoline cycloadducts underwent rearrangement to aziridine derivatives. Expected signals in ${ }^{1} \mathrm{H} \mathrm{NMR},{ }^{13} \mathrm{C}$ NMR, Fourier transformer infrared spectroscopy (FT-IR) were obtained for all the isoxazolidine and isoxazoline derivatives (2-7). Satisfactory elemental analysis values were also obtained for all the novel cycloadducts.

Furthermore, synthetic potential of the novel fluoro isoxazoline derivatives (5-7) are tremendous as they could be converted into 1,3 difunctional amino alcohols (scheme 2). Studies are in progress.

Synthetic potentiality of nitrone $\mathbf{1}$ has been tested successfully as an oxidizing reagent in the conversion of alkyl halides to aldehydes and ketones (scheme 1) following a pattern of atom efficient reactions reported by our group. ${ }^{14}$ Studies are in progress at present in our laboratory. We have already reported synthesis of various aldehydes and ketones from alkyl halides using $\alpha$-chloro nitrones in atom-efficient reactions. ${ }^{14,22}$

\section{Conclusion}

In conclusion, we have shown that 1,3-dipolar cycloadditions of fluoro nitrones with activated alkenes and electron deficient alkynes may be conveniently carried out in RTIL's by obtaining corresponding novel fluoro isoxazolidines and isoxazolines in good conversions and yields with tremendous synthetic potentiality. The ionic liquid may be recycled several times without loss of activity or selectivity.

\section{Supporting Information}

The electronic supporting information can be seen in www.ias.ac.in/chemsci.

\section{Acknowledgements}

We are grateful to the Department of Science and Technology, Government of India, New Delhi for financial support (grant no: SR/S1/OC-34/2011). We are also thankful to the Central Drug Research Institute (CDRI), Lucknow for providing spectral data. 


\section{References}

1. Frederickson M 1997 Tetrahedron $\mathbf{5 3} 403$

2. For a general review of this area see: (a) Tufariello J J 1984 1,3-Dipolar cycloaddition chemistry (ed.) A Padwa (Wiley: New York); (b) Torssell K B G 1988 Nitrile oxides, nitrones and nitronates in organic synthesis (New York: Wiley-VCH)

3. (a) Seerden J P G, Boeren M M M and Scheeren H W 1997 Tetrahedron 53(34) 11843; (b) Gothelf K V and Jørgensen K A 1998 Chem. Rev. 98863

4. Patterson W, Cheung P S and Ernest M J 1992 J. Med. Chem. 35507

5. Wagner E, Becan L and Nowakowska E 2004 Bio. Org. Med. Chem. 12265

6. Confalone P N and Huie E M 1988 Org. React. 361

7. (a) Welton T 1999 Chem. Rev. 99 2071; (b) Wasserscheid P and Keim W 2000 Angew. Chem. Int. Ed. (Engl.) 393772

8. Sheldon R 2001 J. Chem. Soc. Chem. Commun. 2399

9. Gordon C M 2001 Appl. Catal. A: (Gen) 222101

10. Yadav J S, Reddy B V S and Baishya G 2003 J. Org. Chem. 68 7098; (b) Yadav J S, Reddy B V S, Reddy J S S and Srinivas Rao R 2003 Tetrahedron 591599
11. Bach D 1990 Angew. Chem. Int. Ed. (Engl.) 291320

12. Arnone A, Bernardi R, Blasco F, Cardillo R, Gerus I I and Kukhar V P 1998 Tetrahedron 542809

13. (a) Chambers R D 2004 Fluorine in organic chemistry (Oxford, UK: Blackwell Publishing); (b) Kirsch P 2004 Modern fluoroorganic chemistry (Weinheim, Germany: Wiley- $\mathrm{VCH})$

14. Chakraborty B and Sharma P K 2012 Synth. Commun. 421804

15. Chakraborty B, Sharma P K, Rai N, Kafley S and Chhetri M S 2010 J. Chem. Res. 34147

16. Chakraborty B, Sharma P K, Rai N and Sharma C D 2012 J. Chem. Sci. 124679

17. Chakraborty B, Sharma P K and Chhetri M S 2012 J. Heterocyclic Chem. 491260

18. Chakraborty B, Sharma P K and Kafley S 2012 Green Chem. Lett. Rev. 6(2) 141

19. Coskun N and Ozturk 2007 Tetrahedron 631402

20. Deshong P, Li W, Kennington J W and Ammon H L 1991 J. Org. Chem. 56136

21. Gandolfi R and Grunanger P 1999 The chemistry of heterocyclic compounds (New York: Wiley Interscience) 49 774

22. Chakraborty B, Sharma P K, Chhetri M S, Kafley S and Ghosh A R 2011 J. Indian Chem. Soc. 88245 